\title{
USE OF TRAFFIC INTENT INFORMATION BY AUTONOMOUS AIRCRAFT IN CONSTRAINED OPERATIONS
}

\author{
David J. Wing \\ NASA Langley Research Center, Hampton VA \\ Bryan E. Barmore \\ Karthik Krishnamurthy ${ }^{*}$ \\ Titan Systems, Hampton VA
}

Abstract

This paper presents findings of a research study designed to provide insight into the issue of intent information exchange in constrained en-route air-traffic operations and its effect on pilot decision-making and flight performance. The piloted simulation was conducted in the Air Traffic Operations Laboratory at the NASA Langley Research Center. Two operational modes for autonomous flight management were compared under conditions of low and high operational complexity (traffic and airspace hazard density). The tactical mode was characterized primarily by the use of traffic state data for conflict detection and resolution and a manual approach to meeting operational constraints. The strategic mode involved the combined use of traffic state and intent information, provided the pilot an additional level of alerting, and allowed an automated approach to meeting operational constraints. Operational constraints applied in the experiment included separation assurance, schedule adherence, airspace hazard avoidance, flight efficiency, and passenger comfort.

The strategic operational mode was found to be effective in reducing unnecessary maneuvering in conflict situations where the intruder's intended maneuvers would resolve the conflict. Conditions of high operational complexity and vertical maneuvering resulted in increased proliferation of conflicts, but both operational modes exhibited characteristics of stability based on observed conflict proliferation rates of less than 30 percent. Scenario case studies illustrated the need for maneuver flight restrictions to prevent the creation of new conflicts through maneuvering and the need for an improved user interface design that appropriately focuses the pilot's attention on conflict prevention information. Pilot real-time assessment of maximum workload indicated minimal sensitivity to operational complexity, providing further evidence that pilot workload is not the limiting factor for feasibility of an en-route distributed traffic management system, even under highly constrained conditions.

* Member, AIAA $\underline{\text { Introduction }}$

A significant research activity within the NASA Advanced Air Transportation Technologies project is focused upon far-term operations of the National Airspace System (NAS). A general description of the activity is Distributed Air/Ground Traffic Management (DAG-TM). NASA has developed a high-level concept of operations for DAG-TM consisting of 15 elements spanning gate-to-gate operations ${ }^{1}$. One particular concept element, developed to address the en-route flight regime, ${ }^{2}$ has the potential to increase capacity, flexibility, and robustness of the NAS by distributing responsibility for (1) separation assurance, and (2) conformance with local traffic flow management (TFM) constraints between airborne and ground-based systems. In this concept element, pilots of aircraft designated as "autonomous" have the authority to generate and implement new trajectories at their discretion in order to meet individual, company (if applicable), and/or system-level goals. With this authority for autonomous operations comes the responsibility for separation assurance and compliance with local TFM constraints established by the groundbased air traffic service provider (ATSP). Aircraft not operating as autonomous aircraft are designated as "managed aircraft," and similar to current operations, their flight crews comply with clearances provided by the ATSP, who maintains responsibility for their separation assurance and flow management conformance.

\section{$\underline{\text { Research Focus }}$}

\section{Flight-deck Information Requirements}

A predominant research focus in the Free Flight community has been on the type of information required on the flight deck of autonomous aircraft to enable their pilots to ensure separation from other aircraft. Accurate detection of "conflicts" or predicted loss of separation between aircraft is a key requirement for autonomous aircraft operations. At issue are the relative utility and requirement for inter-aircraft information exchange of the current "state" (three dimensional position and velocity vector) and "intent"

$-1-$

American Institute of Aeronautics and Astronautics 
of each aircraft (flight plan); this surveillance information forms the basis for trajectory predictions used in automated conflict detection. Additionally, related human factors issues exist, such as determining how pilots would use the surveillance information, and how this information should be presented on the flight deck displays, considering usability, display design precedence, and integration with other pilot tasks.

Previous research has indicated that, in unconstrained operations (no schedule constraints or airspace restrictions to the route of flight), the exchange of state information between aircraft is sufficient to safely enable airborne self separation in the en route domain $^{3}$. A state-only system has the potential to significantly reduce bandwidth requirements for future surveillance systems such as Automatic Dependent Surveillance - Broadcast (ADS-B), and it reduces the complexity of conformance monitoring and conflict alerting logic. To address the conflict alerts missed by not using intent information, Hoekstra ${ }^{3}$ developed and tested a predictive airborne separation assurance system (PredASAS) that calculates potential off-trajectory conflicts and displays avoidance bands on the heading, airspeed, and vertical speed indicators. This conflict prevention system was designed to provide information regarding which maneuvers would lead to a conflict without the crew needing to "probe" or "try various maneuvers." The conclusion was made that "if all equipped aircraft are fitted with PredASAS, there is no longer a need to know intent information because nobody will turn (or climb/descend) into a conflict." ${ }^{3}$

A study conducted at the NASA Ames Research Center suggested that pilots nevertheless preferred to be provided traffic-aircraft intent information, and the preferred source was Flight Management System (FMS) flight plan data ${ }^{4}$. In this study, flight crews were alternately provided with three types of traffic information: state data, Flight Control Panel (FCP) data, or FMS data. The flight crews were given the opportunity to use voice communication channels to communicate directly with other aircraft to gather intent information or negotiate resolutions. Results of the study indicated that pilot preferences for intent information centered primarily on the improved ability to understand the conflict alerts. Intent information type (state, FCP, or FMS) was found to have no effect on separation assurance.

\section{Operational Constraints}

Whereas these studies addressed unconstrained operations, little research has been performed on the feasibility of constrained operations. Constrained operations are important to consider in concept feasibility and viability analyses. Operational constraints ultimately limit airspace capacity (notwithstanding runway availability limitations), and a concept that does not address capacity limitations is of little practical interest. It is in the more highly constrained conditions that operations will be found to be either fragile or robust to real-world system demands and variability.

Operational constraints can generally be expressed in four categories. Flow management constraints are restrictions that must be imposed to maintain high traffic flow while ensuring safety. Flow management constraints in a future system may include such crossing restrictions as a "required time of arrival" (RTA) assignment at a terminal boundary for inbound autonomous aircraft. Airspace hazard constraints are present when certain regions of airspace are inadvisable for entry. Examples of such airspace hazards are active special-use airspace (SUA) and convective weather cells. Performance constraints include restrictions based primarily on the operating limitations of the aircraft. Restrictions such as maximum operating altitude, speed, or climb/descent rate govern the degrees of freedom available for conflict resolution maneuvers. Economic constraints include user-generated operational guidelines that must generally be met a majority of the time for a commercial aviation business to remain viable for the long term. Examples include fuel efficiency, schedule considerations, and passenger comfort.

When constraints of these types are considered in combination with the task of separation assurance, the type of traffic surveillance information provided to the flight crews may play a more critical role in their ability to repeatedly and reliably meet their separation assurance responsibility than in unconstrained operations. This issue extends beyond the minimum requirement for information that enables airborne separation assurance, and it extends beyond the preferences of the flight crew. The study of constrained operations is critical to determining the overall advisability of exchanging intent information to enable the NAS participants to achieve their operational objectives, meet established constraints, and operate with long-term stability in the future airspace operations.

\section{Modes of Autonomous Operations}

In the experiment, two modes of autonomous aircraft operation, tactical and strategic, that relate to the use of traffic intent information were tested for comparison. The two operational modes are both considered viable solutions, and they differ in several respects beyond just the level of information exchange.

$-2-$

American Institute of Aeronautics and Astronautics 


\section{Tactical mode}

Aspects of the tactical mode have been developed and investigated over several years in batch and piloted simulation studies by the NLR (National Aerospace Laboratory of The Netherlands) ${ }^{3}$, and it is primarily characterized by simplicity in several respects. It was designed to minimize the requirements placed on supporting technology, including both data link and pilot decision-support automation. Broadcast data-link bandwidth requirements are minimized by employing conflict detection based only on the current aircraft state vector (current position, altitude, ground track, ground speed, and vertical speed). On-board conflict detection algorithms deterministically compare (in the current implementation) the state vectors of traffic aircraft with that of the ownship. To minimize false alerts associated with extrapolation errors ${ }^{5}$, state-vectorbased conflict detection is limited in its "look ahead" time horizon. The research of NLR has determined that a five-minute look-ahead horizon is sufficient for separation assurance.

If a conflict is detected, the pilot is alerted, and the conflict resolution algorithm is automatically activated to calculate maneuver advisories for the pilot. These conflict resolution advisories are simple in that they are recommended changes to the ownship heading, airspeed, or vertical speed. The pilot implements the maneuver by setting targets in the FCP to match the advised settings. This procedure is comparable to the pilot's current use of the FCP to comply with a vector for traffic issued by Air Traffic Control (ATC). Concurrently, a conflict prevention system (PredASAS) monitors all possible single-dimensional maneuvers for conflicts, and it indicates to the pilot what maneuvers would cause a new conflict, essentially a "no-go" alerting system. All maneuvers outside of the displayed no-go bands are conflict-free for at least the next 5 minutes, assuming the traffic aircraft do not maneuver during this time

The resolution maneuvers in this operational mode are tactical in nature because they only resolve the conflict and do not account for a return to the original flight plan or the consideration of external constraints, such as RTAs or airspace hazards. This highlights the primary characteristic of the tactical mode, that of the open-loop (manual) nature of meeting constraints. It is hypothesized that the pilot would typically solve problems sequentially: First, resolve the conflict by maneuvering clear; second, avoid any nearby airspace hazards; third, develop an efficient plan to return to course; and fourth, make adjustments to meet RTA and other ATC constraints. This approach has the effect of spreading decision-making over time and possibly simplifying the maneuver decisions. After maneuvering safely to resolve the original conflict, the pilot monitors the conflict prevention information to determine when it is safe to return to course.

\section{$\underline{\text { Strategic Mode }}$}

The strategic mode is a closed-loop (automated) method of trajectory planning. Any trajectory changes recommended to the flight crew will have been determined a priori to meet all known constraints and optimization criteria, while both solving the current conflict and returning the aircraft to course. This approach places greater demand on decision-support automation in that it must generate trajectories for pilot review based on a simultaneous solution of constraints and objectives. More information on future actions of the traffic would allow for earlier detection of many conflict situations and greater flexibility and acceptability of new trajectories. Therefore, the intended trajectory ("intent") of each aircraft is included in its broadcasted data-link message. This approach places a greater demand on the data link bandwidth to accommodate the additional information. For this experiment, the intent message was defined to be a series of trajectory change points, although other forms of intent are also being considered ${ }^{7}$.

Conflict detection in the strategic mode is performed using a combination of traffic state and intent information. The state-based conflict detection is identical to that used in the tactical mode, and a 5minute look-ahead horizon was used in the experiment. The intent-based conflict detection deterministically compared the ownship flight plan to the traffic aircraft broadcast intent in a search for intent conflicts ${ }^{8}$. Whereas a 15-20 minute look-ahead horizon is thought to be appropriate for intent-based conflict detection, a shorter horizon of 8 minutes was used in the current study to allow more data to be gathered during the limited availability of the subject pilots. This shorter time horizon was still of sufficient length to permit exploration of some of the implications of differing intent and state time horizons.

A conflict-alerting decision algorithm was developed to determine when and how to alert the pilot to potential and actual conflict situations. The utility of combining state-based and intent-based conflict detection is that the alerting system can distinguish between a conflict situation that requires ownship action and a situation that will likely be resolved by maneuvers of the traffic aircraft. The latter situation would require no ownship action but would have the potential for elevating to the former category should the traffic aircraft not maneuver appropriately. Such events include failure of the aircraft to observe priority and/or maneuver flight rules (described below) or an unannounced deviation from the broadcast intentions

$-3-$ 
(i.e., flight plan non-conformance). It is hypothesized that distinguishing within the alerting logic between situations that require or do not require ownship action would reduce unnecessary maneuvering and therefore improve overall system stability. The alerting logic is described in more detail in reference 9.

In contrast to the tactical mode, the strategic mode uses modern commercial aircraft technology to assist in conflict resolution. By coordinating the conflictresolution calculations with the flight planning and trajectory generation functions of the FMS, a complete re-planning of the local trajectory can be performed that guarantees that the new trajectory is within the flight envelope. In addition, the FMS can also be used to close the loop on ATSP constraints. Speed and path strategies that meet an RTA at a downstream fix or airspace boundary can be incorporated into the proposed conflict-resolution trajectory. Resolution strategies can also incorporate predicted locations of convective weather cells and scheduled activation of SUA or any region that would be considered hazardous or inadvisable to enter, assuming this information was made available to the aircraft systems. Since the solution space that meets these constraints would normally be large, trajectory optimization can be performed to achieve a desired goal, such as fuel economy, a comfortable ride, or an early arrival. The FMS can then be used to fly the complete resolution trajectory, potentially reducing the task load of the flight crew.

Conflict resolution advisories for conflicts based on valid intent (i.e., the intruder aircraft is determined to be conforming to its broadcast intent) were generated by a genetic-algorithm-based optimization routine ${ }^{10}$. This routine was designed to iterate trajectory constraints with the FMS until a conflict-free trajectory that meets all additional constraints (e.g., RTA) is determined. Further iterations are then performed to optimize a selected parameter (e.g., fuel-burn minimization). The trajectory would normally be flown by the FMS. For conflicts requiring ownship action that are based on state information, resolution advisories identical to those in the tactical mode are presented as a "quick fix" option for the flight crew.

Two types of flight rules are envisioned for the strategic mode of operation, each providing a distinct benefit. A maneuver flight rule is one that governs what types of maneuvers are not permissible in certain situations. The strategic mode incorporates a maneuver flight rule that is designed to prevent near-term conflicts from suddenly appearing. The same rule was applied to the tactical mode, as described earlier in the use of the conflict prevention system. The rule states that an aircraft may not implement a change in track, ground speed, or vertical speed that creates a near-term conflict (for the current study, within 5 minutes). The pilot would meet the requirements of this rule by avoiding flight in the direction of a conflict prevention band, although transition through a band is permitted. This maneuver flight rule has the additional benefit of providing some predictability in autonomous-aireraft operations, which should aid the ATSP in developing stable strategies for managed-aircraft separation.

A priority flight rule defines which aircraft in a given conflict situation is responsible for resolving the conflict. The tactical mode has no priority flight rule in that it assumes every autonomous aircraft shares equal responsibility to resolve conflicts, which is prudent given the limited time horizon for detecting and resolving conflicts. The strategic operational mode also assigns equal responsibility for near-term conflicts. However, for conflicts more than 5 minutes away, the conflict geometry is used to determine who has "rightof-way." By assigning resolution responsibility to one aircraft in a conflict pair, predictability should increase, total maneuvering at the system level should decrease (ideally by one-half since generally only one aircraft in a pair would maneuver), and system-level traffic flow stability may be enhanced. For conflicts detected significantly far in advance (perhaps greater than 15 minutes - a subject of future research), the benefits of assigning responsibility are likely to disappear, and therefore priority flight rules would no longer be applied.

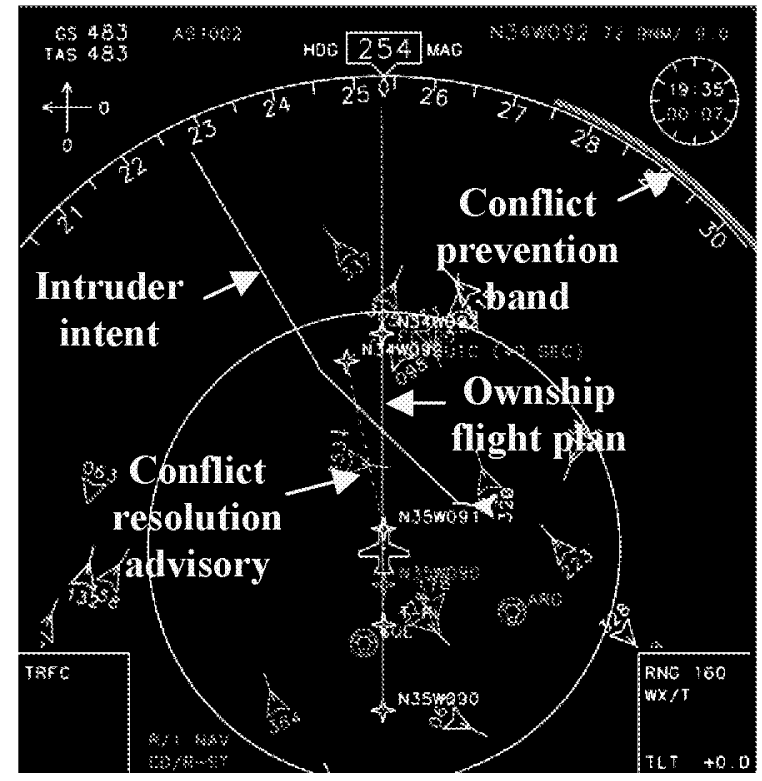

Figure 1. ND as modified to present traffic, conflict, and resolution information. 


\section{Flight Deck Display Design}

A new cockpit display of traffic information (CDTI) design concept, exercised in full in the strategic mode for this experiment, was developed to address the issues of effectively integrating (rather than superimposing) state and intent information for conflict detection into a single presentation. The design built on state-only and intent-only display features previously developed and investigated by $\mathrm{NLR}^{3}$ and NASA Ames Research Center ${ }^{11}$. The aircraft simulation used in the current experiment was a representation of the MD-11 aircraft. The new display features for autonomous operations were therefore integrated into the MD-11 flight-deck display suite, and existing MD-11 conventions were adhered to as much as possible. The Primary Flight Display (PFD) and Navigation Display (ND) were the only displays affected, and an ND control panel was added. The new display design followed the common approach of superimposing traffic data on the ND. The CDTI features are described in reference 9. The ND with some of the CDTI features is shown in figure 1.

\section{Conflict Alerting}

The conflict alerting follows the MD-11 aircraft system alerting convention. The alerting logic is based on three levels of alerting.

A Level 1 alert is used when a conflict situation must be conveyed to the pilot, but no action is required because the intruder aircraft is expected to resolve the conflict. The alert is considered advisory, similar to a traffic "point out." Note that Level 1 alerts only occur in the strategic mode, since it requires priority flight rules and/or traffic intent information to be present.

A Level 2 alert requires action by the ownship flight crew. This alert is used when a conflict has been detected, and it is the responsibility of the ownship flight crew to resolve the situation. A reminder of imminent loss of separation is given to the pilot at 3 minutes to go.

A Level 3 alert requires immediate action by the ownship flight crew. This alert corresponds to the actual loss of separation, i.e., the aircraft have approached within the minimum legal separation criteria.

\section{Conflict Resolution Advisories}

The detection of a conflict triggers the calculation of resolution advisories by the decision support automation. In the tactical mode, maneuver options are displayed to the pilot on the PFD and ND that permit conflict resolution with simple maneuvers (i.e., heading and vertical speed changes). These maneuver options were considered advisories only, and the pilot was free to choose a different solution if desired based on factors not considered by the algorithm (such as other traffic and airspace hazards).

In the strategic mode, a proposed conflict-resolution trajectory is automatically loaded as an alternate route in the FMS and is displayed on the ND for pilot review. A Control and Display Unit (CDU) page was devised for accepting (or rejecting) the trajectory. If the conflict persists until also detected as a state conflict, tacticalmaneuver options (same as those calculated in the tactical mode) are displayed to the pilot to permit immediate conflict resolution for safety. These tactical advisories are shown concurrently with the alternate FMS-route advisory, providing the pilot a tactical option to clear the conflict alert while the strategic FMS route is reviewed.

\section{Experiment Objectives and Approach}

The experiment was conducted in the NASA Langley Air Traffic Operations Laboratory, which contains a medium-fidelity workstation simulation of airspace operations that permits pilots to interact in proposed future ATM environments. A more detailed description of the simulation capability can be found in reference 5 .

The primary objective of the experiment was to compare the two proposed operational modes applicable to airborne separation assurance in a constrained en-route environment. The experiment focused on operational aspects that relate to commercial-transport autonomous aircraft as defined by the DAG-TM Concept Element $5^{2}$.

The experiment focused on the operations of a single autonomous aircraft in en-route cruise flight with variable airspace complexity (i.e., traffic density, weather cells, SUA). Beyond the current research scope were direct interactions with the ATSP, managed aircraft, or other piloted autonomous aircraft. The study did not address multi-person flight crews, crew resource management, or voice communications. Climbs and descents of the ownship were not studied, nor were the effects of winds or failure modes of decision-support automation or CNS infrastructure. These issues will be addressed in future studies.

A 2-by-2-by-3 within-subjects experimental design was used to address the research objectives. The primary independent variables were operational mode (tactical and strategic) and operational complexity (low and high). The third independent variable was the conflict situation design, described below.

Operational complexity, for the purposes of this experiment, was assumed to be a function of traffic density and airspace hazard density. Research has shown that traffic density is correlated with operational

$-5-$

American Institute of Aeronautics and Astronautics 
complexity $^{12}$. Traffic density approximating recorded 1997 levels was used for the low complexity condition and was tripled for the high complexity condition. Airspace hazard density was added as an additional relevant complexity factor of constrained operations, although no conflicts with airspace hazards were planned.

Sixteen active commercial transport pilots participated in the study. Each flew three scenarios in each of the four conditions represented in the 2-by-2 experimental design described above. The level-cruise scenarios each contained one of the following three conflict situations. An "intent-only" conflict situation occurred when only the intent trajectories threatened a conflict. A "state-only" conflict situation occurred when only the state trajectories threatened a conflict (and the intent trajectories did not). A "blunder" conflict situation was similar to the "state-only" conflict situation, but the intruder aircraft did not adhere to the planned trajectory change in the broadcast intent message, and the two aircraft remained in conflict.

Because the ownship did not have access to intent information in the tactical mode, the pilot would have observed a different intruder-aircraft behavior than that planned for each conflict situation. Thus, the pilot's perceptions of the three conflict situations above would differ in the two modes of operation considered in this experiment, resulting in three pilot-perceived conflict types.

Max-alert conflicts: A blunder conflict as defined above loses its meaning when observed in the tactical mode, since the observer (i.e., ownship) never knew about the intruder's planned TCP in the first place. The intruder's behavior would appear to be a "normal" conflict (not precipitated by any intruder maneuver or lack of an expected one) detected at the maximum lookahead time (5 minutes). For the strategic mode observer, the corresponding conflict situation would be the intent-only conflict, which again would be detected at the maximum look-ahead time ( 8 minutes).

Short-alert conflicts: The intent-only conflict observed in the tactical mode is comparable to the blunder conflict observed in the strategic mode, since in both cases the Level-2 conflict alert ("action required") occurred well within the look-ahead horizon (in this study 3.5 minutes were provided by design for both conflict situations). These both could be considered "pop-up" conflicts by their respective observers.

Self-resolving conflicts: The state-only conflict type can be considered directly comparable for tactical and strategic observers, since in both cases the intruder would have appeared to resolve the conflict approximately 1.5 minutes after the alert (as designed into this study). A pilot who took no action would see these conflicts as self-resolving. A pilot who maneuvered may also observe the intruder maneuver and therefore possibly conclude the resolution was shared.

Each scenario was terminated with an RTA constraint at a fix. The subject pilot was tasked to ensure separation from the traffic aircraft and avoid airspace hazards while meeting the RTA constraint. In order to assess workload impact, the subject pilot was given a secondary task involving periodic monitoring and reporting of aircraft system status. Additionally, the pilot was prompted every two minutes to record a real-time assessment of workload on a seven-point scale from very low to very high. Only the latter metric is reported in this paper.

\section{$\underline{\text { Results and Discussion }}$}

Initial findings based on researcher observations of several scenario case studies, pilot subjective ratings and comments on the tactical and strategic operational modes, and pilot subjective ratings of display design features are presented in reference 9. This complementary paper presents a partial analysis of objective numerical trajectory and event data. The discussion of results will focus on the influence of strategic and tactical operations and operational complexity level on maneuvering, conflict proliferation, operational efficiency, and pilot workload. Additionally, case studies are presented on the two scenarios where a loss of separation occurred.

\section{Maneuvering}

As described earlier, each pilot was exposed to three conflict types: max alert, short alert, and self-resolving. The pilot experienced these scenarios in both tactical and strategic modes and in low and high complexity environments. Only two of the three scenarios required a resolution maneuver by the pilot; the scenario containing the self-resolving conflicts would not because the intruder's actions would resolve the conflict approximately 1.5 minutes after the alert. In addition to these planned conflict situations, the pilot encountered additional conflicts (unplanned by the experimenters) as a result of their maneuver choices. The pilots were expected to maneuver to resolve these conflicts as well.

The total count of maneuvers performed in the experiment is presented in Figure 2. When considering all scenarios tested, the maneuver count was relatively insensitive to operational complexity in the strategic mode, but a notable increase in maneuvers was observed in the tactical mode as complexity increased. Note that the metric considers the turn-away and turn-

$-6-$

American Institute of Aeronautics and Astronautics 
back maneuvers as one, and so the effects shown are not indicating differences in the maneuvering procedure. Breaking the maneuver count down by conflict type illustrates that a more complex relationship of maneuvering to operational mode and complexity exists

In the max-alert scenarios, maneuver count was higher in the strategic mode than in the tactical mode, a counter-intuitive result considering that the strategic mode design intended the opposite. Nearly two-thirds of the strategic mode pilot-runs were performed with partial or full tactical maneuvering, even though a pure strategic resolution was always offered. Analysis of the raw data underlying the plot indicates that, in the low complexity case, several pilots chose to second-guess the strategic resolution offered by the automation, increasing the strategic-mode maneuver count. This observation indicates that the maneuver count trend may be the result of inadequate experience with or trust in the strategic resolution advisories, as the pilots had very little time to become familiar with the performance of this tool before the data runs. Additional or alternative factors in the trend may have been conservative decision-making, e.g., providing redundant separation in both the vertical and lateral dimensions, and the absence of strategic vertical resolution capability, as pilots were frequently observed to augment the lateral strategic maneuver with a vertical tactical maneuver. In the high complexity case, the underlying data indicates that more pilots made early (pre-conflict) maneuvers in the strategic mode than in the tactical mode. It is conceivable that the higher complexity caused pilots to maneuver tactically rather than wait for the system to alert them, which points either toward more evidence of inadequate trust and/or experience with the system or possibly a pilot need for a longer look-ahead conflict-detection time in high complexity environments.

In the short-alert scenarios, a mirror-image maneuvercount trend is seen for strategic and tactical modes with respect to an increase in operational complexity; the strategic maneuver count decreases, and the tactical count increases. In the strategic-mode, low complexity scenarios, seven of the maneuvers occurred either during the Level-1 alert ("no action required") or while resolving the primary conflict after returning to course too soon. Both of these may be indicators of a lack of operational experience, but the former may indicate conservative flying tendencies. This data set also included one of the two loss-of-separation pilot-runs, contributing four additional maneuvers to the count. In the tactical mode, a primary contributor to the increase in maneuver count was maneuvers in response to proximate but non-conflicting background traffic; such maneuvers were generally not observed with short-alert

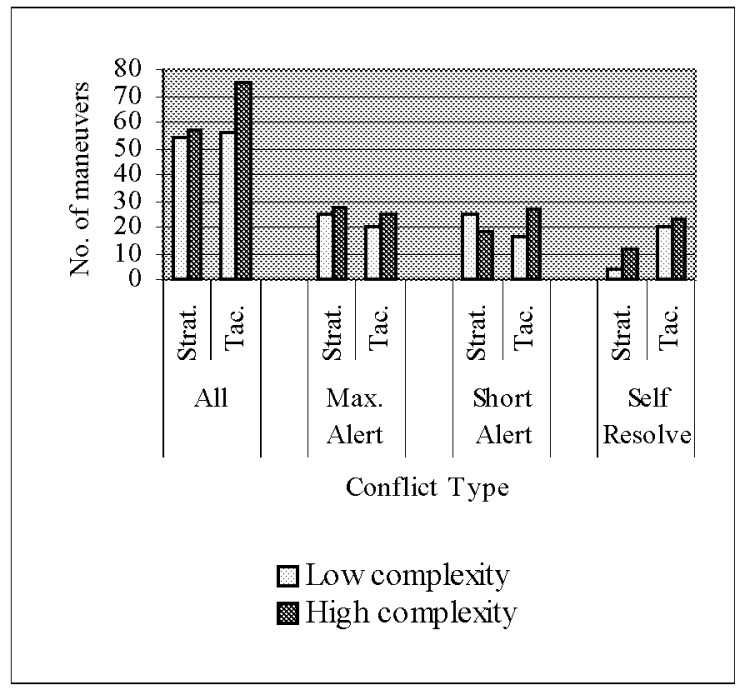

Figure 2. Quantity of maneuvers performed in preventing and resolving conflicts.

conflicts in the strategic mode. It may be that the intent information provided in the strategic mode gave the pilots enough confidence in the future traffic state to forgo preventative maneuvering.

In self-resolving conflict scenarios, strategic mode operations appeared to be effective in reducing unnecessary ownship maneuvers. In this mode, the pilots were informed of the intruder's intent to change trajectory before losing separation, and the Level-1 alert provided the pilot an advisory not to take action. Nearly all recorded maneuvers in strategic mode occurred during this Level-1 alert, the reasons for which were postulated earlier for short-alert scenarios. In most cases, the tactical-mode pilots elected to immediately resolve the conflict upon receiving the alert. Had they waited for the 3-minute warning to maneuver, as they were instructed they could, the conflict would have disappeared with no action by the ownship. The pilots may have considered immediate maneuvering to be the more conservative approach, and yet several new conflicts were created by this decision in the high-complexity scenario, requiring additional maneuvering.

\section{Conflict Proliferation}

The creation of a new conflict as a result of maneuvers to prevent or resolve a previous conflict is clearly an undesired effect. Such conflicts are termed "second generation" in this paper to denote their origin. The more preferred stable traffic environment is one where conflicts do not persist or propagate between aircraft. Requiring the complete elimination of secondgeneration conflicts, however, may be unreasonable. As operational complexity increases, it can be expected

$-7-$

American Institute of Aeronautics and Astronautics 


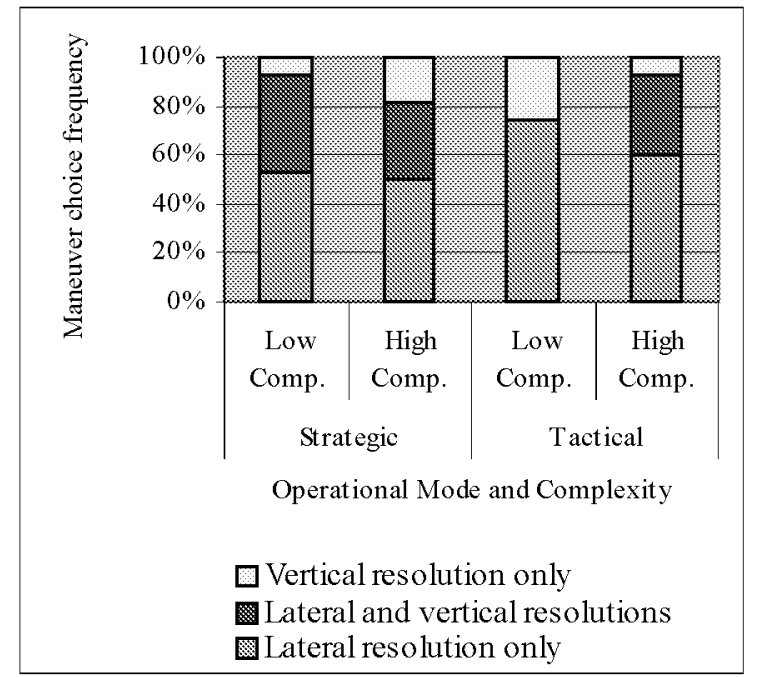

Figure 3. Maneuver choice frequency in intent-only conflict scenarios.

that the unintended creation of new conflicts will occasionally occur, and the vast majority of these will likely occur beyond the ability to detect at the originating maneuver time. A more rare, but still possible, occurrence might arise in highly complex situations where temporarily trading a near-term conflict for a longer-term conflict is the logical or possibly only solution, given other concurrent constraints. Nevertheless, the nominal goal is to minimize the occurrence of second-generation conflicts.

As expected, the preponderance of secondgeneration conflicts in the current experiment occurred in the high-complexity environment, and for reasons unclear, over 50 percent of these occurred in the intentonly conflict type (max-alert scenario in strategic mode; short-alert scenario in tactical mode). In maneuvering to prevent or resolve conflicts, the pilots had the option in both modes to choose between lateral, vertical, or a combined maneuver. Speed was also an option, but is generally considered ineffective as a pure mechanism for conflict resolution in short time domains. As shown in Figure 3, pilots in the intent-only conflict scenarios chose pure lateral maneuvers over vertical or combined lateral/vertical more than half of the time.

The second-generation conflicts in these scenarios, however, only occurred in pilot-runs where vertical maneuvering was involved. As shown in Figure 4, the second-generation conflicts generally occurred when the ownship was either climbing/descending or had previously changed altitude. As shown in Figure 1, a relative-altitude color-coding scheme was used in the display of traffic. Aircraft within $1000 \mathrm{ft}$ of ownship altitude were shown in white and the rest were shown in blue or green. Additionally, an altitude filter was

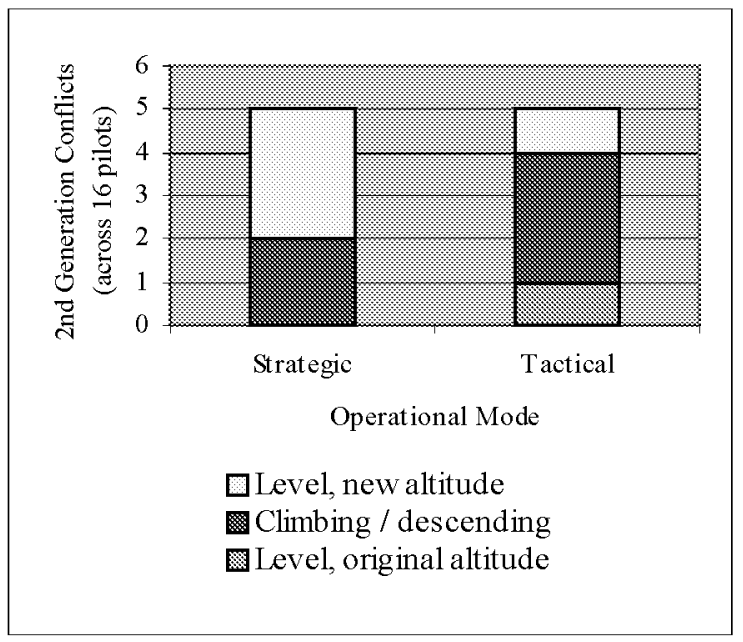

Figure 4. Pilot maneuvering choice in runs involving second-generation conflicts.

available to the pilot for decluttering the display. Both of these features may have contributed to a de-emphasis of aircraft at different altitudes, possibly leading to a loss of awareness of the threat they posed. The fact that many of the second-generation conflicts occurred while climbing or descending indicates that the conflict prevention bands on the vertical speed indicator were not generally in the pilots' scan.

To provide an indication of the stability of the strategic and tactical operations as discussed earlier, the total conflict count in the experiment is presented in Figure 5. As indicated earlier, the majority of the second-generation conflicts occurred in the highcomplexity condition. In the strategic mode scenarios, the 32 planned conflicts resulted in 8 additional secondgeneration conflicts, or a 25 percent proliferation rate. In the tactical mode scenarios, the 31 planned conflicts ( 1 fewer due to an unusable data run) resulted in 9 additional second-generation conflicts, or a 29 percent proliferation rate. Both operational modes, therefore, are reasonably stable at this level of complexity. However, the similarity in proliferation rates between strategic and tactical modes may be a result of the significant use of tactical maneuvering observed in the strategic mode, thereby blurring the true proliferation characteristics of operations in which strategic resolutions are the prevalent maneuver choice. Since data were not obtained at a third complexity level, it is not known whether the proliferation rates vary linearly with increases in complexity.

\section{Operational Efficiency}

In the absence of available fuel-burn data and given the assumptions of the experiment, excess path length

$-8-$

American Institute of Aeronautics and Astronautics 


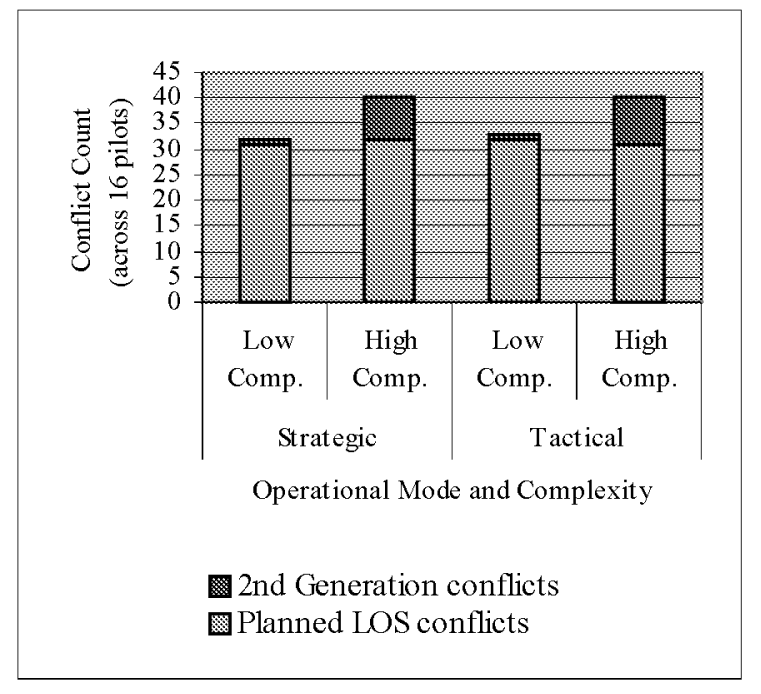

Figure 5. Planned and second-generation conflict count.

flown can be considered a first-order measure of degraded operational efficiency. The initial trajectory of the aircraft in each scenario was a direct constantaltitude path to the RTA fix. Any deviation from this trajectory (to resolve conflicts) may require additional fuel burn and could therefore be considered a reduction in operational efficiency.

Excess path length data is presented in Figure 6. The presented data only includes the pilot-runs involving lateral maneuvers only. Vertical maneuvers generally provide separation with significantly smaller changes in path length and would therefore skew the data when combined with lateral maneuvers. Since the strategic resolution algorithm only provided lateral solutions due to FMS limitations in the current simulation software, the lateral maneuver data is of most interest for comparing strategic and tactical modes. The strategic, maximum-alert data also excludes one particular data point that involved an unusually large lateral deviation compared to the others. The reason for the large maneuver is not known, but may be related to a known guidance problem in the aircraft performance model.

The data in Figure 6 indicate that the strategic and tactical operational modes offered no significant differences in operational efficiency as measured by excess path length. Both modes provided an opportunity to minimize the excess path length in conflict resolution over the current ATC system. In the tactical mode, the pilot could choose to make continual fine-tuning adjustments to minimize flight path deviation from the direct routing, essentially skirting the edge of the required separation criteria, or alternatively the pilot could choose to make fewer gross

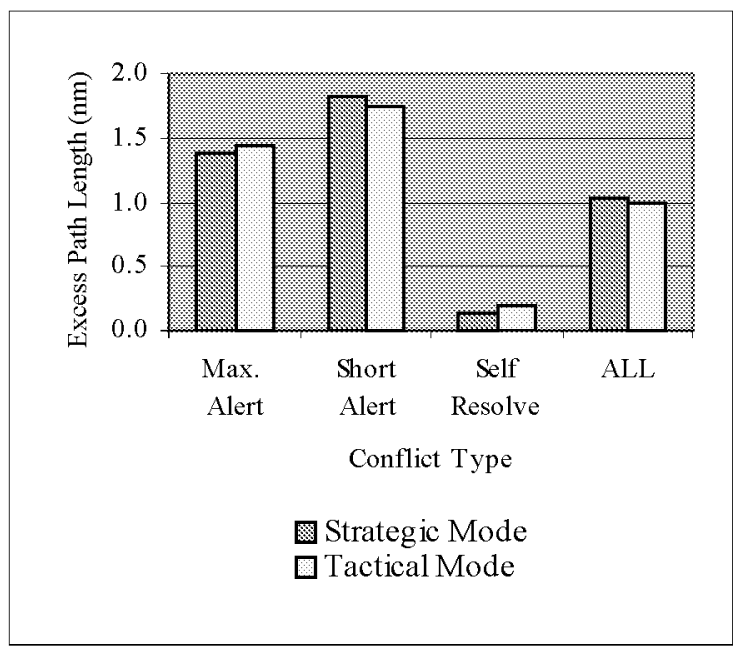

Figure 6. Excess path length for data runs with lateral maneuvers only and strategic resolutions accepted for the maximum-alert scenarios.

changes to the trajectory to minimize pilot task load. In the strategic mode, the automated resolution advisory generated an "optimal" trajectory (based on minimized path length) incorporating a small buffer $(2 \mathrm{~nm})$ to ensure separation, but it involved very few pilot actions to implement. The pilot could choose to have the FMS fly this trajectory or to intervene with tactical maneuvering or a "direct to" modification to the flight plan once the conflict is passed. In the current ATC system, a controller would typically have the goal of providing guaranteed separation with minimal controller workload. Generally, this translates to large buffers and little consideration to optimizing the aircraft's trajectory.

\section{$\underline{\text { Pilot Workload }}$}

As shown in Figure 7, the maximum pilot rating for workload (acquired 8 times during the run on a scale of $1=$ very low, $7=$ =ery high) averaged for the same data set shown in Figure 6 was essentially equivalent in the strategic and tactical modes. This finding is consistent with the observation that the primary contribution to pilot workload was not conflict resolution, but rather scanning and manipulating the ND for traffic information. This task was essentially equivalent in the strategic and tactical modes, except that the strategicmode pilots were also cognitively processing the available intent information. There may be little benefit, and possibly some detriment, to a pilot continuously scanning for possible traffic conflicts when an automated conflict detection system is also performing this task. It may be possible to further reduce pilot workload by eliminating the display of non-threatening aircraft from the ND. A prototype of

$-9-$

American Institute of Aeronautics and Astronautics 


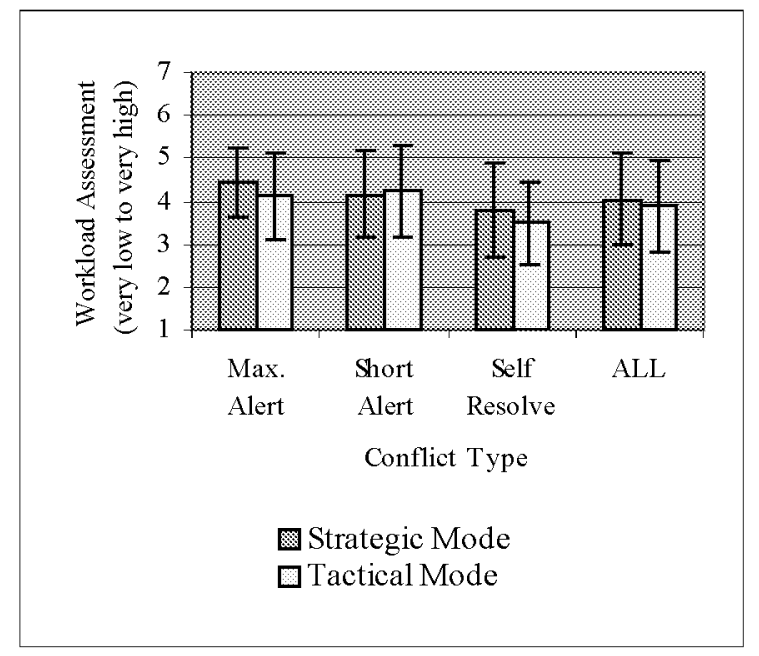

Figure 7. Maximum pilot workload assessments for data runs with lateral maneuvers only and strategic resolutions accepted for the maximum-alert scenarios

such a system will be tested in an upcoming experiment.

The sensitivity of maximum pilot workload assessment on operational complexity is shown in Figure 8 . The data indicate minimal sensitivity of workload to operational complexity. This finding is consistent with the findings of Hoekstra ${ }^{3}$, providing further evidence that an en-route distributed traffic management system may not be limited by pilot workload, as is generally considered to be the case in the current centralized system with controller workload.

\section{Loss of Separation}

Of the 192 scenarios of data acquired in this experiment, two occasions of loss of separation (LOS) occurred, one each in the tactical and strategic operational modes. An LOS for this experiment was defined as coming within 5 nautical miles and 1000 feet of another aircraft, and the conflict resolution advisories and CDTI were designed to assist the pilot in avoiding LOS. It is interesting to note that both LOS situations occurred under conditions of low complexity, which indicates that traffic and airspace hazard density were not likely principal causal factors. It is also interesting to note that the background traffic aircraft were identical for the two LOS scenarios, although the intruder aircraft were different. The actions of one of these background aircraft contributed to both LOS occurrences.

\section{Case 1. Tactical mode}

The designed conflict for this scenario was a stateonly or self-resolving conflict, which means that the intruder's actions would resolve the conflict if the

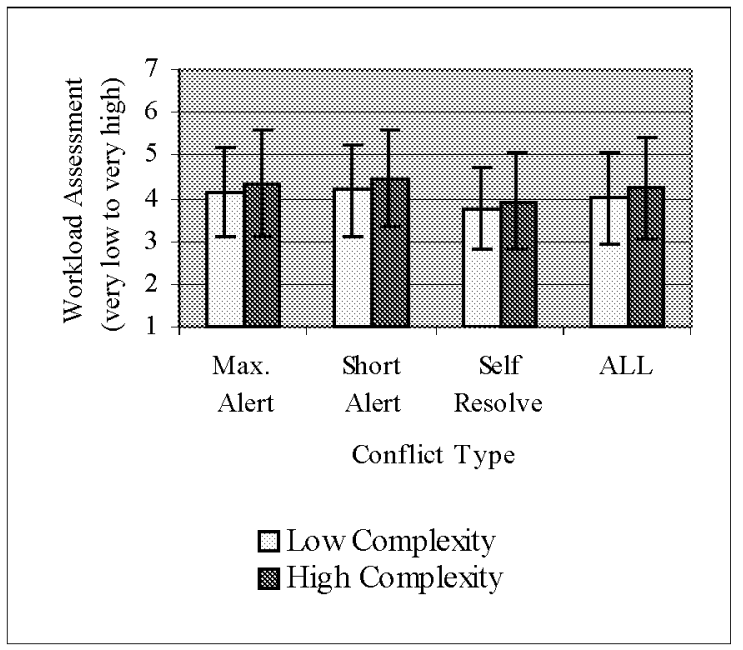

Figure 8 . Sensitivity of pilot workload self-assessment to operational complexity.

ownship were to take no action. In the tactical mode for this conflict type, the ownship pilot has no knowledge this will happen until the intruder initiates the maneuver, which occurs approximately 1.5 minutes after the conflict alert. This case study will illustrate the utility of traffic intent information, the effect of inexperience, and the importance of maneuver flight restrictions.

Synopsis:

The ownship pilot was observing traffic with the ND range set to $80 \mathrm{~nm}$. The four aircraft that were visible were all converging toward the area in front of the ownship. On the left, one aircraft was $4700 \mathrm{ft}$ below ownship and level, and the other was descending through ownship's altitude. The two aircraft on the right were co-altitude with ownship. When the conflict alert appeared for FX689, the planned intruder approaching from the right, the descending aircraft was $2800 \mathrm{ft}$ below. See Figure 9. Although the other approaching aircraft on the right, WN145, was producing a large $\mathrm{CP}$ band for right turns greater than 30 degrees, the ownship pilot turned 20 degrees in that direction, possibly as a turn away from the descending aircraft, TW499. This maneuver momentarily resolved the conflict. The ownship was now navigating towards a 23-degree opening between CP bands. Less than a minute later, two events occurred nearly simultaneously. FX689 started a left turn and WN145 started a right turn, both in the direction of ownship. The turns of both aircraft were part of their unbroadcasted intent, and the result was simultaneous conflicts with the two aircraft. See Figure 10. The 23 degree CP-band opening had collapsed within 25 seconds. The pilot turned right and then corrected hard

$-10-$

American Institute of Aeronautics and Astronautics 


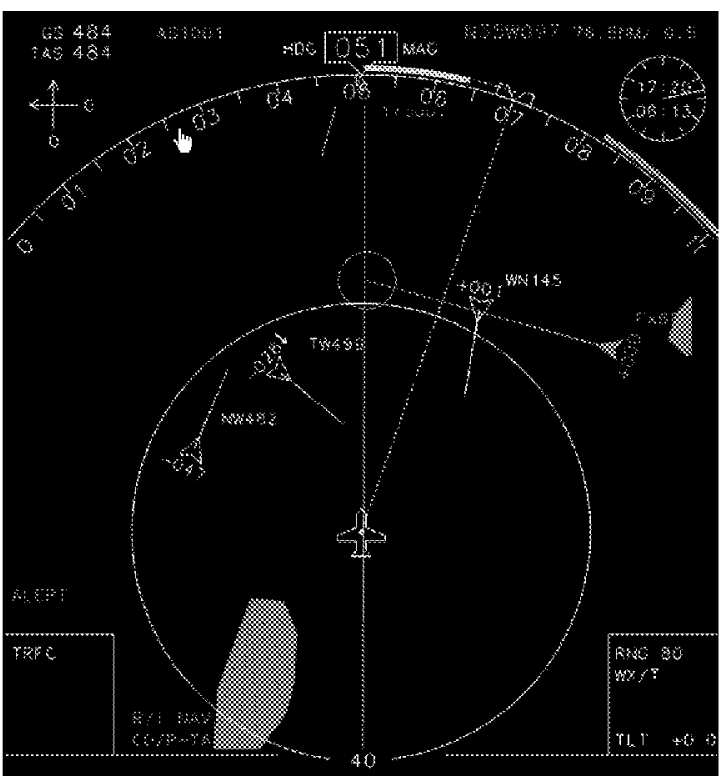

Figure 9. ND screen shot of tactical-mode LOS scenario showing time of first conflict alert.

left (using the FCP since no yoke was provided). Loss of separation occurred during the turn. He regained separation by initiating a climb.

\section{Probable Causes:}

The causal action of the ownship pilot was to turn right to resolve the initial conflict. This eventually led him "into a corner" from which he could not escape with lateral maneuvers. The two aircraft that were nearby, but well below on the left side, may have influenced his decision. This was the pilot's first data run in the experiment, which may indicate that he was still getting used to the displays and/or did not take the time to fully comprehend his situation before maneuvering. Nonetheless, the true causes of the LOS were the turns made by the intruders. By the maneuver flight restrictions in effect, both FX689 and WN145 were prohibited from making turns that result in conflicts. With both aircraft turning, the gap between them closed rapidly, and their proximity to ownship eliminated sufficient time to laterally resolve the conflict. However, these were fully scripted aircraft that did not follow this restriction.

\section{Mitigation:}

More training and experience for the pilot, as well as traffic intent information, may have prevented the initial maneuver that led to the loss of separation. But, this scenario illustrates the requirement for the maneuver flight restriction, which was unequally applied in this experiment due to the scripted traffic aircraft

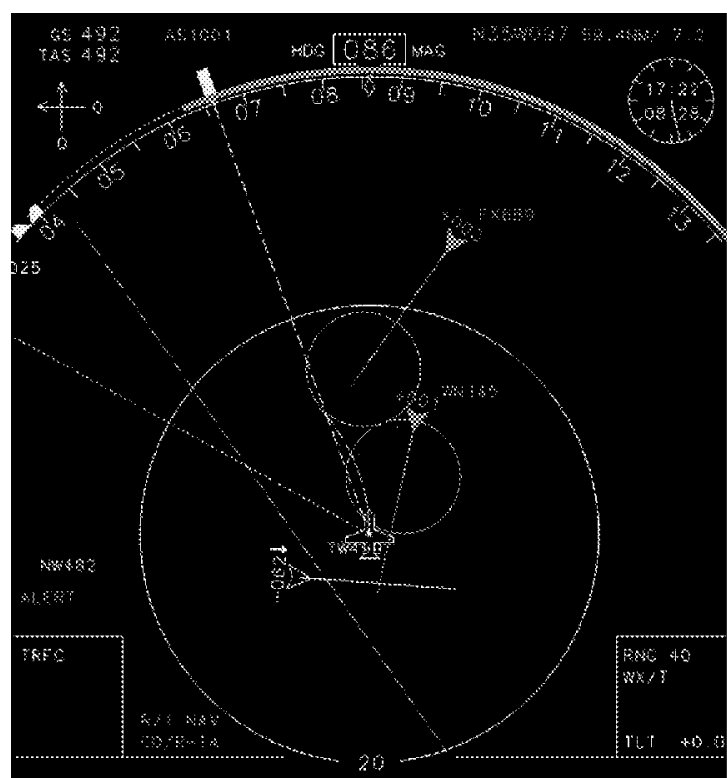

Figure 10. ND screen shot of tactical-mode LOS scenario showing ownship in conflict with two aircraft.

\section{Case 2. Strategic mode}

The designed conflict in this scenario was a vertical blunder, which meant that the intruder was climbing on a conflicting course and broadcasting the intent to level off well below the ownship, but failed to do so. This case study illustrates the profound effect traffic-display declutter features can have on pilot decision-making.

\section{Synopsis:}

The ownship pilot was observing traffic with the ND range set to $80 \mathrm{~nm}$. The altitude filter was set to a large value such that 8 aircraft were shown. About 2 minutes into the 16-minute scenario, the pilot climbed to Flight Level 335 , possibly in response to a perceived threat from DL122 converging from the left and/or WN145 converging from the right. At about 6 minutes into the scenario, a CP band developed on the vertical speed indicator (VSI) indicating a shallow descent would result in a conflict. See Figure 11. About 7 minutes into the scenario, the pilot reset the altitude filter to $+/-3000 \mathrm{ft}$, leaving only WN145 plus 2 other inconsequential aircraft on the ND. Less than a minute later, WN145 started turning towards the ownship, causing the pilot to focus solely on this aircraft, which was $1400 \mathrm{ft}$ below ownship and level. Even though WN145 was about $25 \mathrm{~nm}$ away, the combination of symbol size and ND range setting $(160 \mathrm{~nm})$ made the aircraft appear very close. The pilot reduced the ND range to $10 \mathrm{~nm}$ and then out to $40 \mathrm{~nm}$, and he observed WN145 pass safely behind ownship on the right. Seeing no other aircraft on the ND, the pilot initiated a descent back to Flight Level 320, apparently without 

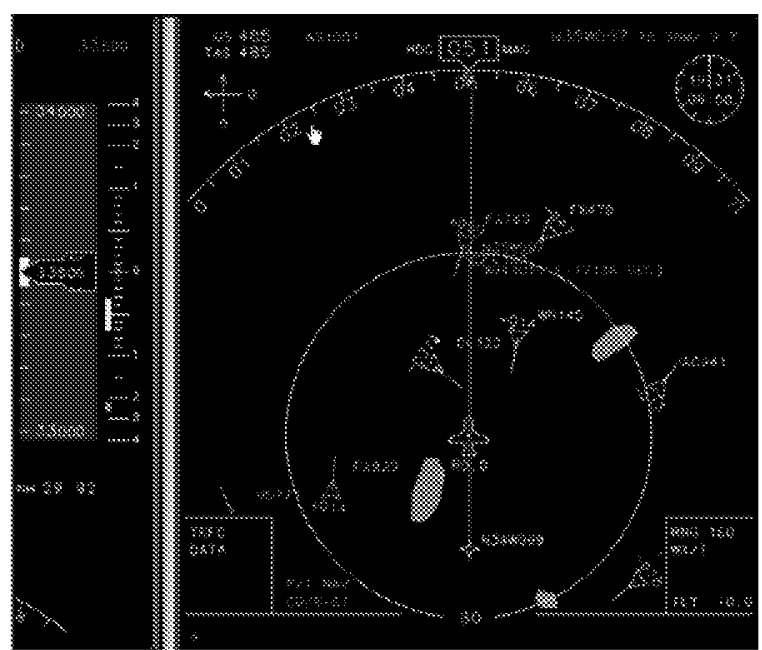

Figure 11. ND screen shot of strategic-mode LOS scenario showing first appearance of conflict prevention band on the VSI.

taking notice of the CP band on the VSI that had been present and slowly growing the whole time. A conflict immediately appeared with the intended intruder, FL520 (AirTran), which was climbing head-on currently $20 \mathrm{~nm}$ in front and $3700 \mathrm{ft}$ below ownship. See Figure 12. The intruding aircraft disappeared and reappeared several times as the ownship vertical speed varied. The pilot initiated a climb to Flight Level 330, but level out occurred within the CP band range, and the conflict reappeared with the intruder about $4 \mathrm{~nm}$ ahead and $1900 \mathrm{ft}$ below. The pilot took no further action, possibly since he predicted he would pass above the intruder and no resolution advisories were presented. LOS occurred as the ownship just clipped the aft corner of the intruder's protected zone.

\section{Probable Causes:}

A principal causal factor was likely the pilot's reduced situation awareness caused by the low altitude filter setting on the ND. The setting allowed the pilot to focus on the safe passage of one aircraft at the expense of knowledge of other proximate aircraft. In the current design, aircraft climbing below the filter limit are not shown. The CP band on the VSI had been present for 4 minutes, but the pilot descended anyway, indicating that he did not take notice of the band when initiating the descent. Had the aircraft causing the band been shown, the pilot might not have descended. Had the intruding aircraft not been scripted, it likely would have contributed to solving the conflict before the LOS. A contributing but less critical factor was the rigorous application of the $1000 \mathrm{ft}$ vertical separation criterion. In level flight, allowance must be made for minor deviations in altitude. For climbing or descending
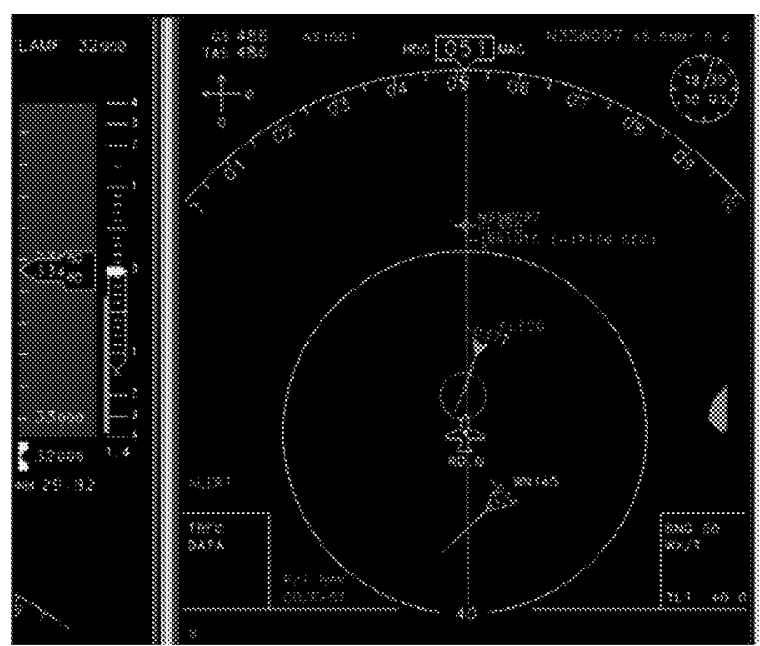

Figure 12. ND screen shot of strategic-mode LOS scenario showing conflict alert after ownship initiated a descent into the conflict prevention band on the VSI.

aircraft, $1000 \mathrm{ft}$ vertical separation may be an inappropriately small separation criterion given the vertical closure rates between aircraft.

\section{Mitigation:}

A redesign of the traffic decluttering features should aid in maintaining the pilot's situation awareness. Although eliminating non-relevant traffic aircraft from the display would likely have significant benefit to the pilot's mental workload, retaining aircraft that pose a potential threat is critical to situation awareness and decision-making.

\section{Conclusions}

In the experimental comparison of strategic and tactical operational modes of autonomous flight management under conditions of low and high operational complexity (defined as the combination of traffic and airspace hazard density), the analyses of trajectory and event data yielded the following conclusions:

\section{Operational Mode}

The strategic operational mode is effective in reducing unnecessary maneuvering in conflict situations where the intruder's intended maneuvers would resolve the conflict.

The pilots' observed reluctance to rely solely on the fully automated lateral conflict resolution advisories were possibly influenced by lack of experience with the tool, conservative decision-making in the absence of experience-based decision-making, and the unavailability of automated vertical resolution capability in the simulation. These factors illustrate the 
challenge in human-in-the-loop testing of advanced DAG-TM concepts, where pilots cannot yet rely on operational experience and fully capable tools in making decisions in a complex autonomous flight management environment.

Both the strategic and tactical operational modes exhibit characteristics of stability based on observed conflict proliferation rates of less than 30 percent. The relative stability between the two operational modes was indeterminate because the pilots in the experiment made limited use of pure strategic resolutions.

No significant differences in operational efficiency as measured by excess path length were observed between strategic and tactical operational modes.

The process of scanning and manipulating the ND for traffic information, a nearly equivalent task in strategic and tactical modes as tested, represents a significant component of pilot workload, and is a redundant function where automated conflict prevention and detection tools are provided. A redesign of the user interface to de-emphasize continual traffic scanning is recommended and may possibly provide benefits by reducing workload and enhancing maneuver decision-making while maintaining safety.

Operational Complexity

Conditions of high operational complexity result in the occurrence of second-generation conflicts, which are defined as conflicts created as a result of earlier conflict prevention or resolution maneuvers. Although a large increase in occurrences were seen over the low complexity condition, insufficient data was acquired to determine the nature of the relationship with respect to increasing operational complexity (e.g., linear, step function). Vertical maneuvering increases the likelihood of second-generation conflicts over lateral maneuvering. Improvements in traffic display and alerting design should target the reduction of secondgeneration conflicts by improving conflict prevention information relative to vertical maneuvers

Pilot real-time assessment of maximum workload indicated minimal sensitivity to operational complexity. This finding provides further evidence that pilot workload is not the limiting factor for feasibility of an en-route distributed traffic management system even under highly constrained conditions.

The two scenarios that contained a loss of separation illustrate the need for maneuver flight restrictions in the operational concept to prevent the creation of new conflicts through maneuvering and the need for an improved user interface design that appropriately focuses the pilot's attention on conflict prevention information.

\section{$\underline{\text { Concept Feasibility }}$}

Within the scope and limits of the design and implementation of this HITL experiment, the results of the investigation support a finding of feasibility for autonomous aircraft operations in the "En Route Free Maneuvering" concept element of the DAG-TM operational concept. Whereas additional challenges to concept feasibility remain and have yet to be fully explored, no insurmountable impediments were found. The experimental results, in fact, provide encouraging evidence that a distributed traffic management system can be devised that is effective in a constrained operational environment.

\section{Acknowledgements}

The following persons are recognized and appreciated as having made substantial contributions to experiment design, simulation and laboratory development, and/or data processing and analysis: Mark Ballin, Richard Barhydt, Tom Britton, Frank Bussink, Alan Campbell, Brian Davis, Wim den Braven, Chace Hall, Bart Heesbeen, Jacco Hoekstra, Tom Laird, Ian MacLure, Stephane Mondoloni, Mike Palmer, Mark Peters, Lisa Rippy, Nicole Sacco, John Schade, and Ari Stassart.

\section{$\underline{\text { References }}$}

1. NASA Advanced Air Transportation Technologies Project: Concept Definition for Distributed Air/Ground Traffic Management (DAG-TM), Version 1.0. September 1999.

2. Phillips, Charles T. Detailed Description for (DAG-TM) Concept Element 5 "En Route Free Maneuvering", Contract NAS2-98005 Research Task Order 41, Advanced Air Transportation Technologies Project, NASA Ames Research Center, 2000.

3. Hoekstra, Jacco M.: Designing for Safety the Free Flight Air Traffic Management Concept. ISBN 90806343-2-8, Doctoral Thesis, Delft University of Technology, November 2001.

4. Cashion, P. \& Lozito, S. The effects of different levels of intent information on pilot self-separation performance. Proceedings of International Symposium on Aviation Psychology. 1999

5. Mondoloni, Stephane; Palmer, Michael T;; and Wing, David J.: Development of a Prototype Airborne Conflict Detection and Resolution Simulation Capability. AIAA-2002-4446, August 2002. 
6. Kuchar, J. K. and Yang, L.C.: Survey of Conflict Detection and Resolution Modeling Methods, AIAA-97-3732, August 1997.

7. Barhydt, Richard and Warren, Anthony W. Development of Intent Information Changes to Revised Minimum Aviation System Performance Standards for Automatic Dependent Surveillance Broadcast (RTCA / DO-242A). NASA/TM-2002211669, May 2002.

8. McDonald, James A.; Vivona, Robert A.: Strategic Airborne Conflict Detection of Air Traffic and Area Hazards, Version 1.0. NASA Contract: NAS2-98005 RTO-29, November 6, 2000.

9. Wing, D. J.; Adams, R. J.; Duley, J. A.; Legan, B. M.; Barmore, B. E., and Moses, D.: Airborne Use of Traffic Intent Information in a Distributed Air-Ground Traffic Management Concept: Experiment Design and Preliminary Results. NASA/TM-2001-211254, November 2001.

10. Mondoloni, Stephane; Conway, Sheila R.: An Airborne Conflict Resolution Approach Using a Genetic Algorithm. AIAA-2001-4054, August 2001.

11. Johnson, Battiste, and Bochow: Enabling Strategic Flight Deck Route Re-Planning Within A Modified ATC Environment: The Display of 4-D Intent Information on a CSD. SAE 2000-01-5573, 2000.

12. Mogford, R.; Guttman, J.; Morrow, S.; and Kopardekar, P.: The Complexity Construct in Air Traffic Control: A Review and Synthesis of the Literature. DOT FAA Technical Report, DOT/FAA/CT-TN95/22. July 1995.

$-14-$

American Institute of Aeronautics and Astronautics 